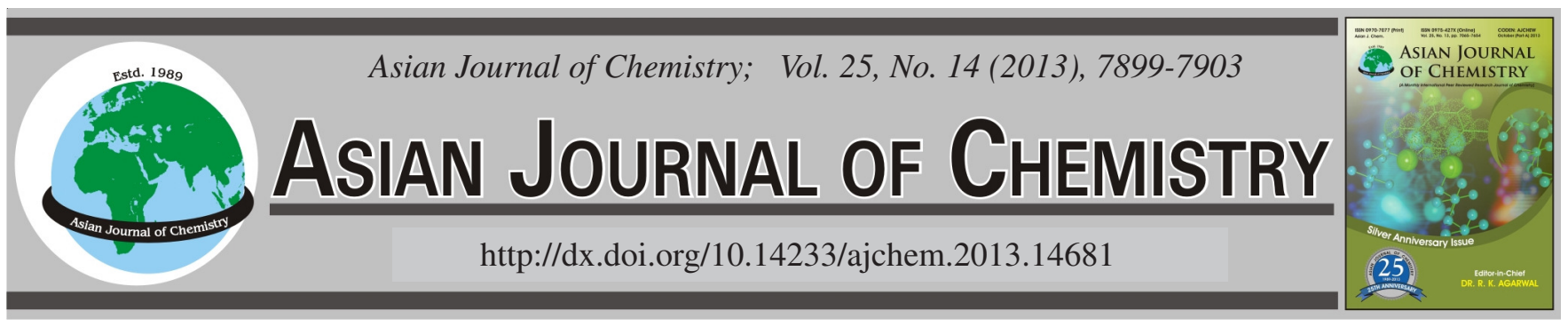

\title{
Characterization of the Constituents Absorbed in Rats After Oral Administration of Fructus Gardeniae Effective Fraction
}

\author{
Xiaojuan Wang ${ }^{1,3}$, Zhixiong $\mathrm{LI}^{1}$, Bin Wu ${ }^{1}$, Yujing $\mathrm{ShI}^{2}$, Shanshan Guo ${ }^{2}$, Yubin JI ${ }^{3}$, Xiaolan CuI ${ }^{2, *}$ and ChengGang Huang ${ }^{1, *}$
}

\author{
${ }^{1}$ Shanghai Insitute of Materia Medica, Chinese Academy of Sciences, Shanghai 201203, P.R. China \\ ${ }^{2}$ Institute of Chinese Medicine, China Academy of Chinese Medical Sciences, Beijing 100029, P.R. China \\ ${ }^{3}$ Harbin University of Commerce, Harbin 150076, P.R. China \\ *Corresponding authors: Tel: +86 21 20231963; E-mail: cghsimm@126.com; 493984443@163.com
}

\begin{abstract}
Fructus gardeniae (Chinese herbal name is $\mathrm{Zhi} \mathrm{Zi}$ ) is widely used as a traditional Chinese medicine (TCM) for treating various diseases. The research discovered the iridoid glycosides that extracted and isolated from fructus gardeniae have antiviral activity. However, its bioactive constituents have remained unknown. In the present study, a systematic method based on high-performance liquid chromatography coupled with electrospray ionization mass spectrometry (HPLC-MSn) techniques was developed to identify the absorbed constituents in rats after oral administration of target fraction. By comparing their MS data with those of authentic compounds and published data, a total of five compounds [deacetyl asperulosidic methyl ester (1); gardenoside (2); scandoside methyl ester (3); genipin gentiobioside (4) and geniposide(5)] were identified in the target fraction. In addition, we identified five iridoid glucosides and one of their metabolite in rat urine samples, four iridoid glucosides and one of their metabolite in rat serum samples after administration, respectively. This provides an accurate profile of the composition of bioactive compounds responsible for the antiinfluenza properties and builds a solid bottom for future research on its mechanism.
\end{abstract}

Key Words: Fructus gardeniae, HPLC-MSn, Iridoid glycosides, Urine, Serum.

\section{INTRODUCTION}

Gardenia is a popular ornamental shrub that can be found worldwide. The fruits of Fructus gardeniae is widely used as a traditional Chinese medicine (TCM) in many Asian countries for its antiphlogistic, cholaneresis, sedative and antipyretic effects $^{1,2}$. The major effective constituents of Gardenia fruits are iridoid glycosides, flavonoids, crocetins, etc. ${ }^{3,4}$. Gardenia extracts have antiviral activities against Herpes Simplex Virus-1 and Parainfluenza Virus- 1 in vivo and in vitro ${ }^{5,6}$, but the effective compounds are not to be found.

Traditional chinese medicine are increasingly drawing attention as alternative treatment approaches and latent sources of antiviral drugs due to the existence of many secondary metabolites with interesting biological activities ${ }^{7,8}$. However, in contrast to target-oriented Western medicine, traditional Chinese medicine uses a holistic and synergistic approach to restore the balance of Yin-Yang of body energy so that the body's normal function and homeostasis can be restored ${ }^{9,10}$. Nevertheless, the effective ingredients of traditional Chinese medicine are too complicated to be identified by traditional methods like pure compound isolation and structural clarification. The effective constituents of traditional Chinese medicine formulas can potentially be ascertained by analyzing the compounds absorbed in the blood after oral administration, since the compounds that absorbed in the blood are likely to exert curative effects ${ }^{11}$, so we could trace the target compounds which could be detected in blood ${ }^{1}$. Therefore, the powerful precise instruments, such as liquid chromatography coupled with mass spectrometry are useful tools for the identification of component structure. Because of its high sensitivity and strong ability of structural exposition, it plays a crucial role in component analysis of which absorbed in the blood ${ }^{12,13}$. Thus, new methods for activity evaluation and molecular target identification of such a multi-component medicine are extremely needed to advance the modernization of traditional Chinese medicine.

In this research, we extracted and isolated iridoid glycosides from Gardenia extracts. The target fraction was given to rats orally and the blood and urine were collected. Then a sensitive and specific LC-ESI-MSn method was applied to identify the compounds absorbed into biological samples.

\section{EXPERIMENTAL}

Fructus gardeniae was purchased from the Kangqiao Medicine Co. in November 2011 and identified by Professor 
Chenggang Huang, principle investigator at Shanghai Institute of Materia Medica, Chinese Academy of Sciences. NKA-9, X-5 macroporous resin (Tianjin Nankai Hecheng Technology Co., Ltd.). Standard geniposide and gardenoside were purchased from the National Institute for food and drug Control (Beijing, China). All standards's purities were above $98 \%$ determined by HPLC. Genipin gentiobioside and scandoside methyl ester were isolated from Fructus Gardeniae and its purities were $>$ $95 \%$ determined by HPLC-UV analysis.

HPLC-grade acetonitrile and methanol were obtained from Dikma Co. (Lake Forest, CA, USA). All other chemicals and solvents were of analytical-grade reagents and were purchased from the Sinopharm Chemical Reagent Co. (Shanghai, China). Distilled water, prepared from demineralized water, was used throughout the experiment.

Extraction and isolation: About $2 \mathrm{~kg}$ of dried fruit of Gardenia jasminoides Ellis was chopped and extracted with distilled water $(2 \times 20 \mathrm{~L})$ for $2 \mathrm{~h}$ every time. After filtration, the extract was combined and evaporated to $20 \mathrm{~L}$ at $70{ }^{\circ} \mathrm{C}$ under reduced pressure. At room temperature, sample solution passed through a glass chromatography column $(8 \mathrm{~cm} \times 80$ $\mathrm{cm}$ ) that was filled with the handled NKA-9 macroporous resin at acertain flow rate in order to remove most of the pigment impurities. Then the effluent was placed in the handled X-5 resin column $(8 \mathrm{~cm} \times 80 \mathrm{~cm})$ and absorbed at the speed of 1 $\mathrm{BV} / \mathrm{h}$ and washed with distilled water $(6 \mathrm{~L})$ until colourless then eluted with $30 \%$ ethanol to give 8 fractions of $2 \mathrm{~L}$ each and the fractions were combined according to the HPLC analysis results. Fractions 3-5 were combined and concentrated to 100 $\mathrm{mL}$ by rotary vaporization at $70{ }^{\circ} \mathrm{C}$ under reduced pressure and then vacuum dried (YX, $8.5 \mathrm{~g}$ ).

Instrumentation and conditions: The identification of compounds was performed by the comparison of the characteristics of the ultraviolet spectra, retention time of standard compounds, molecular weights and fragmentation ions in the documents. The assay was performed on G6310 ion-trap mass spectrometer (Agilent Technologies, Palo Alto, CA, USA), equipped with an electrospray ionization system and controlled by Agilent LC/MSD trap software. MS data were acquired using negative electrospray as the ionization process and the MS/MS data were acquired in automatic mode. A high-speed bench-top centrifuge (TGL-16 C, Shanghai Anting Scientific Instrument Factory, Shanghai, China) was used to separate the biological specimen, which were extracted by a $\mathrm{C}_{18}$ solid phase extraction (SPE) cartridge (1 mL/100 mg; Supelco, USA).

The Agilent 1200 HPLC was equipped with a reversedphase column (Agilent Eclipse XDB-C ${ }_{18}(4.6 \mathrm{~mm} \times 250 \mathrm{~mm}$, $5 \mu \mathrm{m})$ and preceded by an EasyGuard KitC18 $(4 \mathrm{~mm} \times 2 \mathrm{~mm})$ guard column. A model Agilent 1200 HPLC system, consisting of a vacuum degasser, binary solvent delivery pump, autosampler and thermostated column compartment (Agilent) was used to acquire chromatograms. The column temperature was kept constant at $25^{\circ} \mathrm{C}$. Detection wavelengths were set at $238 \mathrm{~nm}$. The flow rate was $1 \mathrm{~mL} \mathrm{~min}^{-1}$. A gradient elution of $0.5 \%$ aqueous acetic acid (A) and acetonitrile (B) was used as 5-10 $\% \mathrm{~B}$ at $0-8 \mathrm{~min}, 10-15 \% \mathrm{~B}$ at $8-30 \mathrm{~min}, 15-40 \% \mathrm{~B}$ at $30-40$ $\min , 40-100 \% \mathrm{~B}$ at $40-45 \mathrm{~min}, 100 \% \mathrm{~B}$ at $45-50 \mathrm{~min}$ and $5 \%$ $\mathrm{B}$ at 50-60 min. The mass spectra were recorded in negative modes, drying gas flow rate $10 \mathrm{~L} \mathrm{~min}^{-1}$, drying gas temperature $35^{\circ} \mathrm{C}$, nebulizer 35 psig, capillary voltage $4,000 \mathrm{~V}$, fragmentor $100 \mathrm{~V}$, mass range of $\mathrm{m} / \mathrm{z} 50-1,500$.

Animals: All animal treatments were strictly in accordance with protocols approved by the Review Committee of Animal Care and Use at the Shanghai Institute of Materia Medica (Shanghai, China). Eighteen male Sprague-Dawley rats (200220 g; Shanghai SLAC Laboratory Animal Co., Shanghai, China) were acclimatized to the facilities for one week before starting the experiments. The animals were divided into three groups at random: group $\mathrm{A}(\mathrm{n}=6)$ for pre-dose samples (i.e., blanks) collection; group B $(n=6)$ for post-dose blood sample collection; and group $C(n=6)$ for post-dose urine sample collection. All animals were fasted (but with access to water) for $12 \mathrm{~h}$ prior to oral administration of $400 \mathrm{mg} / \mathrm{kg}$ doses of YX.

Sample preparation: Blood samples were collected at $0.5,1,2,3,4.5$ and $6 \mathrm{~h}$ after dosing and centrifuged at 12,000 $\mathrm{g}$ for $10 \mathrm{~min}$ to obtain the plasma. Samples of urine was collected and combined, during $0-24 \mathrm{~h}$ post-administration. All the samples were stored at $-80^{\circ} \mathrm{C}$ for future analysis. All the preparation procedures of plasma and urine samples were according to Liu et al. ${ }^{14}$ and Chen et al. ${ }^{15}$. Then, $600 \mu \mathrm{L}$ samples were loaded onto an SPE column (1 mL capacity, $100 \mathrm{mg}$ ODS, Supelco, USA) which was then prearranged with $2 \mathrm{~mL}$ methanol and $2 \mathrm{~mL}$ water subsequently. Then the cartridge was washed with $2 \mathrm{~mL}$ water and the analytes were eluted with $1 \mathrm{~mL}$ methanol. The eluent was dried under nitrogen gas over a water bath of $37^{\circ} \mathrm{C}$. The residues were reconstituted in $200 \mu \mathrm{L}$ methanol and centrifuged twice at $12,000 \mathrm{~g}$ for $10 \mathrm{~min}$. Aliquots of 20 $\mu \mathrm{L}$ of the supernatants were injected into the HPLC-MSn for analysis. Blank samples as control were processed in the same method as drug-containing samples.

\section{RESULTS AND DISCUSSION}

Identifications of constituents in YX: Efficient separation of different components in YX was achieved using reversephase HPLC. Based on the retention time, UV spectrum and MS/MS spectra, 5 bioactive compounds were tentatively identified. Compounds 2, 3, 4, 5 were unambiguously identified as gardenoside, scandoside methyl ester, genipin gentiobioside and geniposide, respectively, by comparison of their mass fragmentation behaviour and retention time with standards, whereas the others were identified based on their UV absorption and MS data. The MS spectra of the characteristic components detected in YX are shown in Fig. 1.

For the compound $\mathbf{5}\left(\mathrm{t}_{\mathrm{R}}=24.1 \mathrm{~min}\right)$ represented the main constituent of $G$. jasminoides. It exhibited $\left[\mathrm{M}+\mathrm{CH}_{3} \mathrm{COO}\right]^{-}$ ion at $\mathrm{m} / \mathrm{z} 447$ and $[\mathrm{M}-\mathrm{H}]^{-}$ion at $\mathrm{m} / \mathrm{z}$ 387. In addition, MS/ MS fragmentation of the molecular ion at $\mathrm{m} / \mathrm{z} 387$ produced an ion with the peak at $\mathrm{m} / \mathrm{z} 225\left([\mathrm{M}-\mathrm{H}-162]^{-}\right)$, indicating the existence of a glucose. Its MS/MS data was consistent with geniposide ${ }^{16}$. Thus, compound $\mathbf{5}$ was identified as geniposide, which was confirmed by comparison with a pure standard.

For the compound $4\left(\mathrm{t}^{\mathrm{R}}=18.6 \mathrm{~min}\right)$ gave a $[\mathrm{M}-\mathrm{H}]^{-}$ion at $\mathrm{m} / \mathrm{z} 549$ and showed almost identical MS/MS spectra (Table-1). It was probably the same aglycone as with geniposide, Based on the retention time of standard, compound $\mathbf{4}$ was identified as genipin gentiobioside. 


\begin{tabular}{|c|c|c|c|c|}
\hline \multicolumn{5}{|c|}{$\begin{array}{c}\text { TABLE-1 } \\
\text { DETERMINATION BY HPLC/DAD/ESI-MSn OF THE COMPOUNDS DETECTED } \\
\text { IN RAT URINE AFTER THE ORAL ADMINISTRATION OF THE YX }\end{array}$} \\
\hline No. & $\begin{array}{l}\text { Time } \\
(\mathrm{min})\end{array}$ & $\begin{array}{l}\text { Parent ion } \\
(\mathrm{m} / \mathrm{z})\end{array}$ & HPLC-ESI-MSn (m/z) & Identification \\
\hline 1 & 11.4 & 807 & $\mathrm{MS}^{2}$ [807]: 403,241; $\mathrm{MS}^{3}[241]: 193,139,101 ; \mathrm{MS}^{4}[139]: 121$ & Deacetyl asperulosidic methyl ester \\
\hline 2 & 12.4 & 403 & $\operatorname{MS}^{2}$ [403]: $371,241,223,193 ; \operatorname{MS}^{3}[241]: 223,193,127$ & Gardenoside \\
\hline 3 & 13.1 & 807 & $\mathrm{MS}^{2}[807]: 403,241 ; \mathrm{MS}^{3}[241]: 193,139,101 ; \mathrm{MS}^{4}[139]: 121$ & Scandoside methyl ester \\
\hline 4 & 18.6 & 549 & $\mathrm{MS}^{2}[549]: 387,323,225,179 ; \mathrm{MS}^{3}[225]: 207,123$ & Genipin gentiobioside \\
\hline 5 & 24.1 & 447 & $\operatorname{MS}^{2}$ [447]: $387,283,225 ; \mathrm{MS}^{3}[225]: 179,123$ & Geniposide \\
\hline 6 & 10.5 & 565 & $\operatorname{MS}^{2}[565]: 403,241,193$ & $\begin{array}{l}\text { Metabolite of glucose conjugation of deacetyl } \\
\text { asperulosidic methyl ester or scandoside methyl } \\
\text { ester }\end{array}$ \\
\hline
\end{tabular}
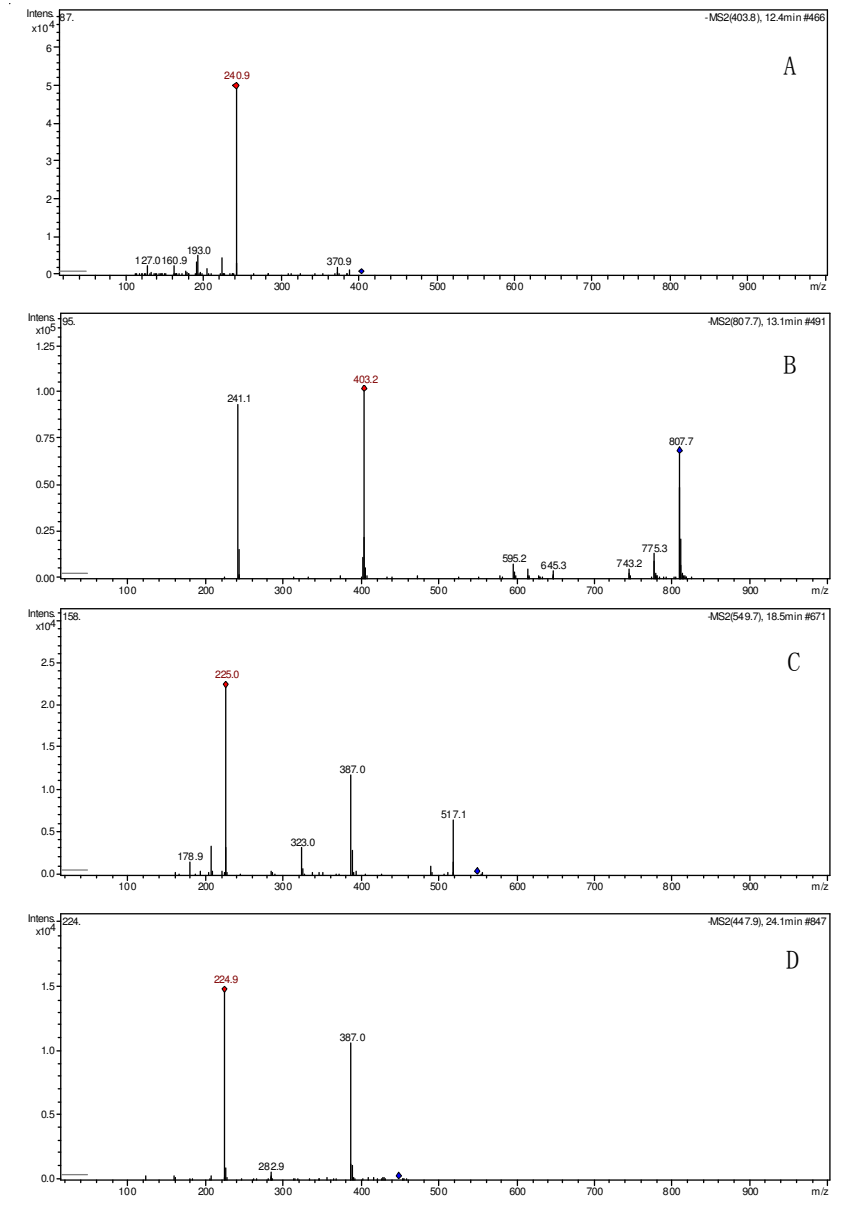

Fig. 1. MS spectra of the characteristic components detected in FR-1: gardenoside (A), scandoside methyl ester (B), genipin gentiobioside (C), geniposide (D)

For the compound $2\left(\mathrm{t}_{\mathrm{R}}=12.4 \mathrm{~min}\right)$ exhibited a $[\mathrm{M}-\mathrm{H}]^{-}$at $\mathrm{m} / \mathrm{z}$ 403. Its MS/MS spectrum showed [M-H-32] $]^{-}$at m/z 371, [M-H-162] $]^{-}$at 241, [M-H-180] $]^{-}$at 223 and [M-H-162-48] $]^{-}$at 193. Meanwhile, compound $\mathbf{1}\left(t_{R}=11.3 \mathrm{~min}\right)$ and $\mathbf{3}\left(t_{R}=13.1\right.$ $\mathrm{min}$ ) had the same $[\mathrm{M}-\mathrm{H}]^{-}$ion at $\mathrm{m} / \mathrm{z} 403$ and $\left[\mathrm{M}+\mathrm{CH}_{3} \mathrm{COO}\right]^{-}$ ion at $\mathrm{m} / \mathrm{z} 463$. The deprotonated molecular ion $[\mathrm{M}-\mathrm{H}]^{-}$yielded four fragments at $\mathrm{m} / \mathrm{z} 283,241,139$ and 101 on the MS/MS spectra, which represented different diagnostic fragmentations. From the published data, gardenoside as well as deacetyl asperulosidic methyl ester and scandoside methyl ester provided the same $[\mathrm{M}-\mathrm{H}]^{-}$ion at $\mathrm{m} / \mathrm{z} 403$ in accordance with the molecular formula $\mathrm{C}_{17} \mathrm{H}_{24} \mathrm{O}_{11}$. Based on these analysis of fragmentation behaviours in the ESI-MS/MS and description of previous documents ${ }^{17}$, compound 2 was identified as gardenoside, whereas compound $\mathbf{1}$ and $\mathbf{3}$ was identified as deacetyl asperulosidic methyl ester and scandoside methyl ester.

Identification of the compounds detected in rat urine samples after oral administration of YX: By comparison of the total ion current chromatogram of the blank rat urine samples with those of the rat urine samples collected during the time interval 0-24 $\mathrm{h}$ after oral administration of the YX, it was shown that the information from the urine samples collected during 0-24 h was the most abundant. The total ion current chromatograms of the blank rat urine and the rat urine samples collected between $0-24 \mathrm{~h}$ after the oral administration of YX are shown in Fig. 2. Six compounds, corresponding to compounds 1-6 in the total ion current chromatogram (Table-1), including five YX components and one metabolite, were tentatively elucidated as compounds 2, 3, 4, 5, 1 (deacetyl asperulosidic methyl ester) and $\mathbf{6}$ (metabolite of glucose conjugation of deacetyl asperulosidic methyl ester or scandoside methyl ester).

For the compound $6\left(\mathrm{t}_{\mathrm{R}}=10.5 \mathrm{~min}\right)$ gave a $[\mathrm{M}-\mathrm{H}]^{-}$ion at $\mathrm{m} / \mathrm{z} 565$ and $[\mathrm{M}-\mathrm{H}-162]^{-}$ion at $\mathrm{m} / \mathrm{z}$ 403. Its MS/MS spectrum showed [M-H-162-162] $]^{-}$at 241, which was probably the same aglycone as with deacetyl asperulosidic methyl ester or scandoside methyl ester. So compound 6 was tentatively identified as metabolite of glucose conjugation of deacetyl asperulosidic methyl ester or scandoside methyl ester.

For the compound $\mathbf{1}\left(\mathrm{t}_{\mathrm{R}}=11.4 \mathrm{~min}\right)$ exhibited a $[\mathrm{M}-\mathrm{H}]^{-}$at $\mathrm{m} / \mathrm{z} 403$ and $\left[\mathrm{M}+\mathrm{CH}_{3} \mathrm{COO}\right]^{-}$ion at $\mathrm{m} / \mathrm{z} 463$. The deprotonated molecular ion $[\mathrm{M}-\mathrm{H}]^{-}$yielded four fragments at $\mathrm{m} / \mathrm{z} 241,193$, 139 and 101 on the MS/MS spectra, which was same with scandoside methyl ester. Based on these analysis of fragmentation behaviours in the ESI-MS/MS and description of previous documents ${ }^{18}$, compound $\mathbf{1}$ could be characterized as deacetyl asperulosidic methyl ester.

Identification of the compounds detected in rat serum samples after oral administration of YX: By comparison of the total ion current chromatograms of the blank rat serum with the rat serum samples collected at $0.5,1,2,3,4.5$ and 6 $\mathrm{h}$ after oral administration of $\mathrm{YX}$ solution, it was shown that the information in the chromatogram at $1 \mathrm{~h}$ post administration was the most abundant. The total ion current chromatograms of the blank rat serum and the rat serum sample collected at $1 \mathrm{~h}$ after the oral administration of YX solution are shown in Fig. 3. Five compounds, corresponding to compounds $\mathbf{1 - 5}$ in the total ion current chromatogram (Fig. 3 and Table-2), including four YX components and one metabolite, were tentatively elucidated as compounds $\mathbf{2 , 3 , 4 , 5}$ and $\mathbf{1}$ (genipin gentiobioside deglycosylation). 

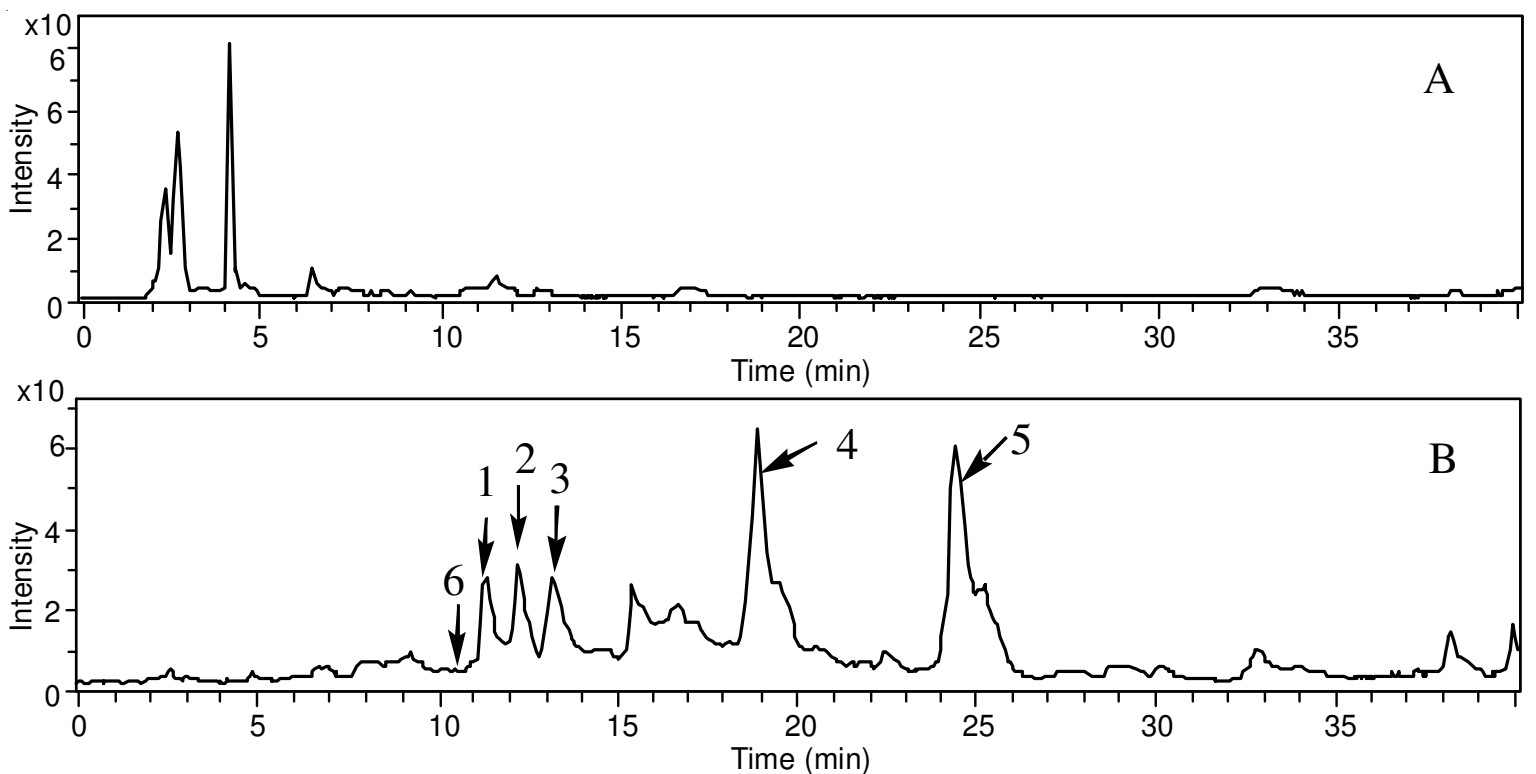

Fig. 2. Total ion current chromatograms of (A) the blank rat urine sample and (B) the urine sample collected during the time interval $0-24 \mathrm{~h}$ after oral administration of the YX. The labeled chromatographic peaks were identified by MS as follows: peak 1, deacetyl asperulosidic methyl ester; peak 2, gardenoside; peak 3, scandoside methyl ester; peak 4, genipin gentiobioside; peak 5,geniposide; peak 6, metabolite of glucose conjugation of deacetyl asperulosidic methyl ester or scandoside methyl ester
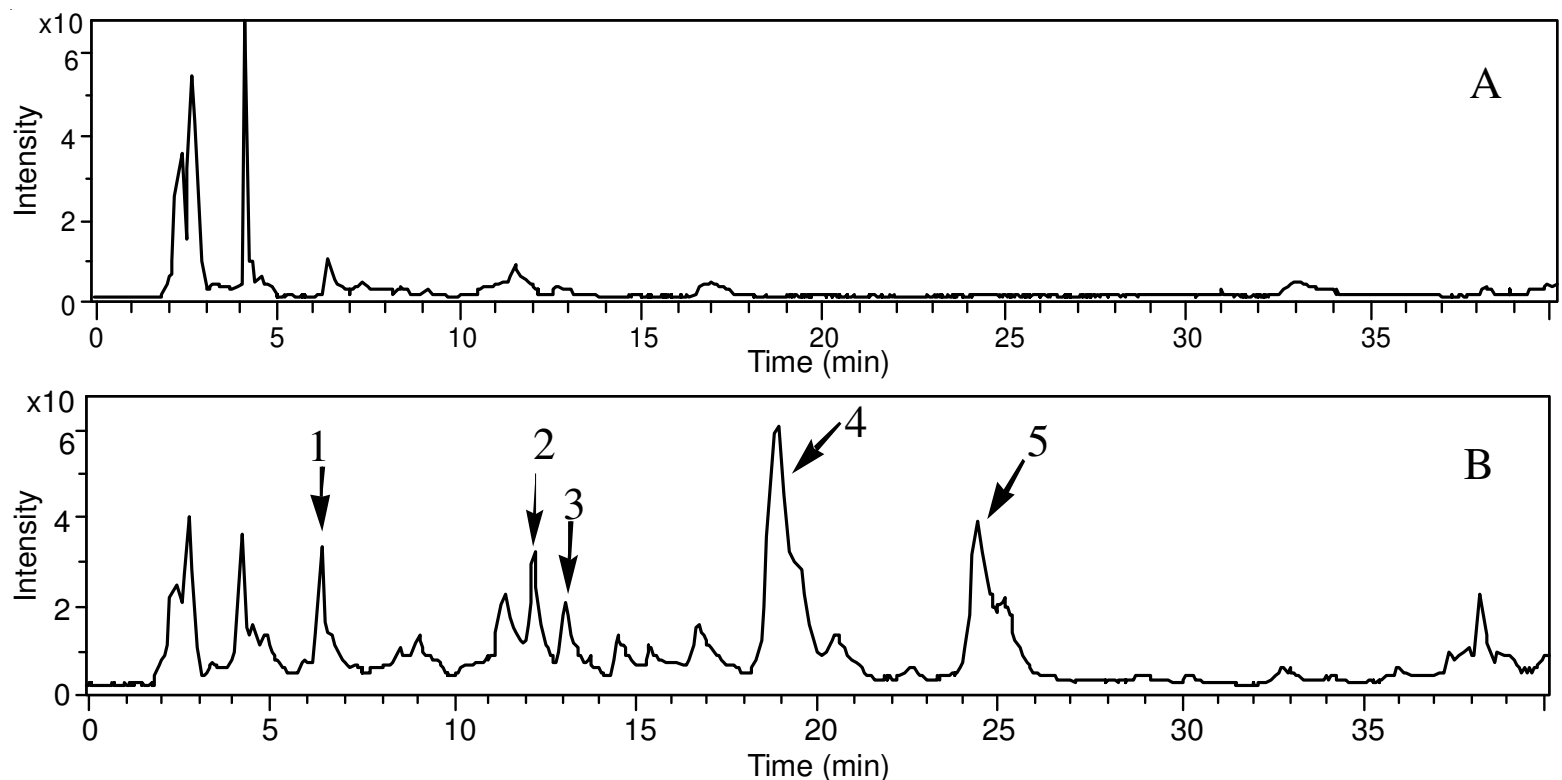

Fig. 3. Total ion current chromatograms of (A) the blank rat serum sample and (B) the serum sample collected at $1 \mathrm{~h}$ post administration of the YX. The labeled chromatographic peaks were identified by MS as follows: peak 1, deglycosylation product of genipin gentiobioside; peak 2, gardenoside; peak 3, scandoside methyl ester; peak 4, genipin gentiobioside; peak 5, geniposide

\begin{tabular}{|c|c|c|c|c|}
\hline \multicolumn{5}{|c|}{$\begin{array}{c}\text { TABLE-2 } \\
\text { DETERMINATION BY HPLC/DAD/ESI-MSn OF THE COMPOUNDS DETECTED } \\
\text { IN RAT SERUM SAMPLE AFTER ORAL ADMINISTRATION OF THE YX }\end{array}$} \\
\hline No. & Time (min) & Parent ion $(\mathrm{m} / \mathrm{z})$ & HPLC-ESI-MSn (m/z) & Identification \\
\hline 1 & 6.2 & 387 & $\mathrm{MS}^{2}[387]: 225,123 ; \mathrm{MS}^{3}[225]: 207,123$ & Genipin gentiobioside deglycosylation \\
\hline 2 & 12.6 & 403 & $\operatorname{MS}^{2}[403]: 241,223,193 ; \operatorname{MS}^{3}[241]: 223,193,127$ & Gardenoside \\
\hline 3 & 13.4 & 463 & $\operatorname{MS}^{2}[463]: 403,241,139 ; \mathrm{MS}^{3}[241]: 139,101$ & Scandoside methyl ester \\
\hline 4 & 18.6 & 549 & $\operatorname{MS}^{2}[549]: 517,387,225,207,179 ; \mathrm{MS}^{3}[225]: 207,123$ & Genipin gentiobioside \\
\hline 5 & 24.2 & 447 & $\operatorname{MS}^{2}[447]: 387,285,225,207 ; \operatorname{MS}^{3}[225]: 123,101$ & Geniposide \\
\hline
\end{tabular}

The MS2 spectra of the compound 1 molecular ion at $\mathrm{m} / \mathrm{z} 387$ exhibited $\left([\mathrm{M}-\mathrm{H}]^{-}\right)$signals at $\mathrm{m} / \mathrm{z} 207,123$, which was the same as the MS3 spectra of $\mathrm{m} / \mathrm{z} 549 \rightarrow 387$ (loss of a neutral fragment of $162 \mathrm{Da}$ ). Therefore, peak 1 could be characterized as the deglycosylated product of genipin gentiobioside.

\section{Conclusion}

In recent years, there has priority of using natural plant products for preventing and treating viral infections. In the present research, we applied HPLC-DAD-ESI- MSn to separate and characterize the compounds in rat urine and serum 
samples after oral administration of the effective fraction, a total of $\mathbf{6}$ and $\mathbf{5}$ compounds were detected, respectively. The four compounds were detected in the rat urine and serum samples simultaneously. These constituents were 2 (gardenoside), 3 (scandoside methyl ester), 4 (genipin gentiobioside) and $\mathbf{5}$ (geniposide; Fig. 4). Otherwise, we identified one prototype 1 (deacetyl asperulosidic methyl ester) and one metabolite of glucose conjugation of deacetyl asperulosidic methyl ester or scandoside methyl ester in rat urine and one metabolite of genipin gentiobioside, genipin gentiobioside deglycosylation in rat serum.

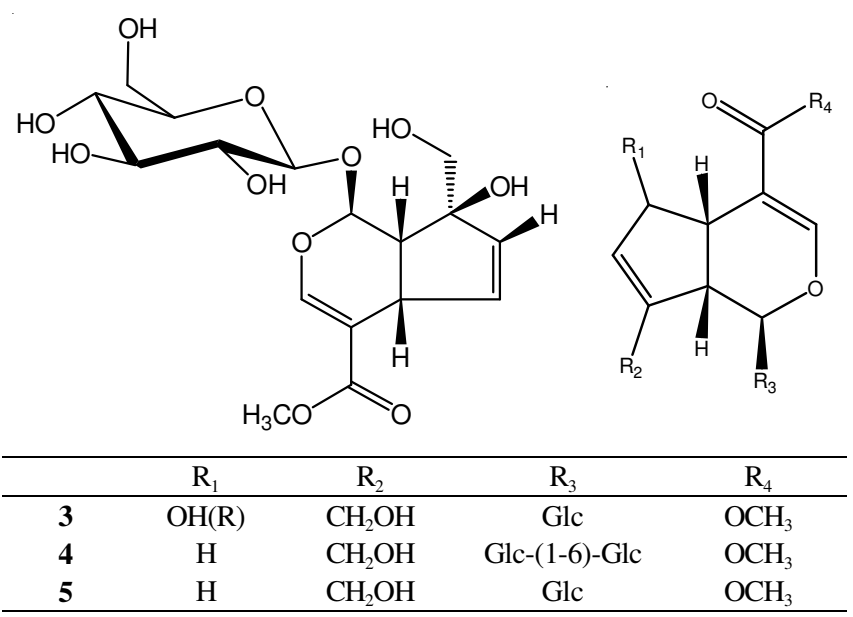

Fig. 4. Chemical structures of the absorbed constituents in rats after oral administration of the YX. 2, gardenoside; 3, scandoside methyl ester; 4 , genipin gentiobioside; 5 , geniposide

Since the constituents absorbed into blood and sustaining at a concentration level in target organs can possess effects abilities to cure diseases. Hence, these compounds might be responsible for the the anti-influenza virus potential of $G$. jasminoides. Our results significantly narrow the extent of effective constituents to be identified and played a solid bottom for further research of the mechanism of potential active components.

\section{ACKNOWLEDGEMENTS}

The authors thank the National Science \& Technology Major Project "Key New Drug Creation and Manufacturing Program" (Nos. 2012ZX09301001-001, 2009ZX09103-373) for financial support of this work.

\section{REFERENCES}

1. J.S. Tian, Y.L. Cui and L.M. Hu, Neurosci. Lett., 479, 236 (2010).

2. Y. Yu, Z.L. Xie and W.W. Ma, Nat. Prod., 72, 1459 (2009).

3. T.T. Zhou, W.Q. Zhao, G.R. Fan, Y.F. Chai and Y.T. Wu, J. Chromatogr. B, 858, 296 (2007).

4. Y. Yu, Z.L. Xie, H. Gao, W.W. Ma, Y. Dai, Y. Wang, Y. Zhong and X.S. Yao, Nat. Prod., 72, 1459 (2009).

5. Y.Z. Wang, X.L. Cui, Y.J. Gao, S.S. Guo, X.K. Wang, Y. Huang, Y. Zhao and W.F. Gong, Zhongguo Zhong Yao Za Zhi, 31, 1176 (2006).

6. S.S. Guo, Y. Huang, Y. Zhao, Y.J. Gao, W.F. Gong and X.L. Cui, Bing Dи Хие Bao, 23, 384 (2007).

7. J.E. Forero, L. Avila, N. Taborda, P. Tabares, A. López, F. Torres, W. Quiñones, M.A. Bucio, Y. Mora-Pérez, M.T. Rugeles, P. Joseph-Nathan and F. Echeverri, Phytochemistry, 69, 2815 (2008).

8. W.J. Shin, K.H. Lee, M.H. Park and B.L. Seong, Microbiol. Immunol., 54, 11 (2010).

9. T. Efferth, P.C. Li, V.S. Konkimalla and B. Kaina, Trends Mol. Med., 13, 353 (2007).

10. Z. Wen, Z. Wang, S. Wang, R. Ravula, L. Yang, J. Xu and C. Wang, PLoS One, 6, e18278 (2011).

11. C.H. Ma, L.X. Wang, Y.H. Tang, M.S. Fan, H.B. Xiao and C.G. Huang, Biomed. Chromatogr., 22, 1066 (2008).

12. X. Wang, W. Sun, H. Sun, H. Lv, Z. Wu, P. Wang, L. Liu and H. Cao, J. Pharm. Biomed. Anal., 46, 477 (2008).

13. Z.H. Peng, W. Song, F.M. Han, H.X. Chen, M.M. Zhu and Y. Chen, Int. J. Mass Spectrom., 269, 131 (2008).

14. W.D. Zhang, X.L. Yang, L. Xu, J. Cao, P. Li and Z.L. Yang, Biomed. Chromatogr., 23, 219 (2008).

15. H.H. Liu, K. Wang, Y.H. Tang, Z.L. Sun, L.H. Jian, Z.X. Li, B. Wu and C.G. Huang, J. Pharm. Biomed. Anal., 55, 1075 (2011).

16. L.Y. Chen, Z.X. Li, Y.H. Tang, X.L. Cui, R.H. Luo, S.S. Guo, Y.T. Zheng and C.G. Huang, J. Pharm. Biomed. Anal., 56, 382 (2011).

17. L. Ren, X. Xue, F. Zhang, Y. Wang, Y. Liu, C. Li and X. Liang, Rapid. Commun. Mass Spectrom., 21, 3039 (2007).

18. T. Zhou, H. Liu, J. Wen, G. Fan, Y. Chai and Y. Wu, Rapid., Commun. Mass. Spectrom., 24, 2520 (2010). 